\title{
AN EFFICIENT ALGORITHM FOR ANALYSIS OF NONLINEAR HEAT TRANSFER WITH PHASE CHANGES
}

\author{
W. DONALD ROLPH III $\dagger$ AND KLAUS-JÜRGEN BATHE $\ddagger$ \\ Department of Mechanical Engineering, Massachusetts Institute of Technology, Cambridge, Massachusetts, U.S.A.
}

\begin{abstract}
SUMMARY
A new, simple and effective finite element procedure is presented for the practical solution of heat transfer conditions with phase changes. In this method, a fixed finite element mesh is employed, and a relatively coarse finite element mesh and large time step can be used in the incremental solution. The results of various numerical studies using the algorithm are presented that demonstrate the effectiveness of the procedure.
\end{abstract}

\section{INTRODUCTION}

Finite element analyses of heat transfer processes can be valuable in various areas of engineering design. In this paper we are concerned with the analysis of temperature distributions and heat flow when phase changes take effect. A problem of this kind is also referred to as the Stefan problem. ${ }^{1}$ Practical applications are encountered in the analysis of the formation of ice, permafrost conditions and welding problems. ${ }^{1-3}$

The basic difficulty in the finite element modelling of heat transfer problems with phase changes lies in that the transformation between phases is accompanied by either absorption or liberation of latent heat in the phase transition zone. The physics of the problem leads to a temperature solution with discontinuous temperature gradients at the phase transition surface. To date, several authors have used analytical, finite difference and finite element methods to analyse such problems, ${ }^{1-12}$ but for practical analysis the use of a finite element method appears to be most promising. Of the procedures presented, the technique of Ichikawa and Kikuchi ${ }^{11,12}$ is attractive because of its stability and accuracy characteristics. A disadvantage of the method is that it requires the use of a freezing index as the state variable, instead of temperature, which means that the procedure cannot easily be added to existing conventional finite element computer programs for heat transfer analysis.

A method that can directly be employed in conventional finite element computer programs is the procedure proposed by Morgan et al. ${ }^{9}$ However, this technique must be used with care, because for a given phase change temperature interval $\Delta \theta_{f}$ the time step used must be small enough that the change in temperature during one time step, in a region undergoing a change of phase, is less than $\Delta \theta_{f}$. Hence, the procedure cannot be used in the analysis of a pure substance for which $\Delta \theta_{f}$ is zero, and may require an unrealistically small time step in a practical solution.

The objective in this paper is to present an algorithm that is very simple and effective for the analysis of nonlinear temperature conditions with phase changes. In the procedure we use

$\dagger$ Research assistant.

$\ddagger$ Associate Professor.

0029-5981/82/010119-16\$01.60

Received 8 October 1980

(C) 1982 by John Wiley \& Sons, Ltd. 
a fixed finite element mesh and relatively large time steps. Restrictions on the time step size and mesh configurations are greatly reduced by comparison with previously published methods, and no special conditions on the phase change temperature interval need be satisfied. Moreover, the procedure is easily implemented in conventional finite element computer programs for nonlinear heat transter analysis.

In this paper we first summarize the governing equations to be solved and then present the details of our algorithm for solution. Finally, we give the results of some sample solutions that demonstrate the effectiveness of the proposed solution technique.

\section{GOVERNING EQUATIONS, BOUNDARY AND INITIAL CONDITIONS}

The equation of conservation of energy considered in this study may be written in a Cartesian space as

$$
-\partial q_{i} / \partial x_{j}+q^{B}=\rho c \dot{\theta}
$$

where

$$
q_{i}=-k_{i j} \partial \theta / \partial x_{j}
$$

and $\theta$ is the temperature, $k_{i j}$ are conductivity coefficients. The variables $\rho, c$ and $q^{B}$ are the mass density, the specific heat and the rate of heat generation per unit volume, respectively. It should be noted that in equation (1) the thermal properties can be a function of position and temperature.

Considering a three-dimensional body, see Figure (1), the following boundary conditions may be specified:

Specified temperature. The temperature can be prescribed at a specific surface of the body, denoted by $S_{\theta}$ in Figure (1).

Specified heat flux. The heat flow input may be prescribed at specific points or surfaces of the body

$$
q^{S}=q_{i} n_{i}
$$

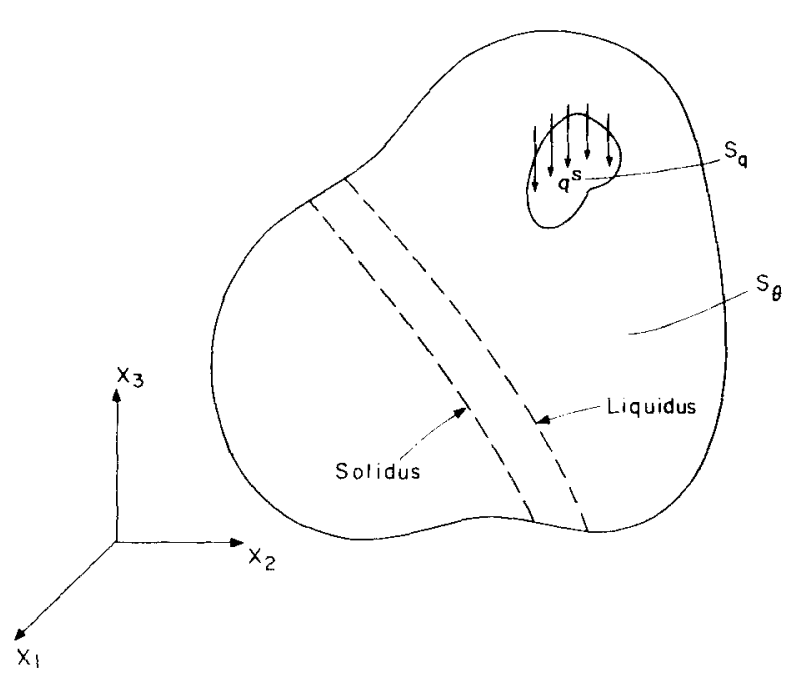

Figure 1. Three-dimensional body under heat transfer conditions 
where $q^{S}$ is the heat flow input on surface $S_{q}$ and the $n_{i}$ are the components of a unit vector n normal to the surface $S_{q}$.

Convection boundary conditions. Considering convection boundary conditions we have

$$
q^{S}=h\left(\theta_{e}-\theta^{S}\right)
$$

and $h$ is the convection heat transfer coefficient, which may be temperature-dependent, and $\theta_{e}$ and $\theta^{S}$ are the environmental and surface temperatures, respectively.

Radiation boundary conditions. Considering radiation boundary conditions we have

$$
q^{S}=\kappa\left(\theta_{r}-\theta^{S}\right)
$$

where $\theta_{r}$ is the temperature of the external radiation source and $\kappa$ is a radiative coefficient,

$$
\kappa=h_{r}\left(\theta_{r}^{2}+\theta^{S^{2}}\right)\left(\theta_{r}+\theta^{S}\right)
$$

The variable $h_{r}$ is determined from the Stefan-Boltzmann constant, the emissivity of the radiant and absorbing materials and the geometric view factors.

Phase change interfaces. In the case of a phase change (of a pure substance), the following boundary conditions must be satisfied at the phase transition interface:

$$
\left.\begin{array}{l}
\theta=\theta_{f} \\
\Delta q^{S} \mathrm{~d} S= \pm \rho L \frac{\mathrm{d} V}{\mathrm{~d} t}
\end{array}\right\} \text { on } S_{f}
$$

where $\theta_{f}, \rho, L$ are the phase change temperature, mass density and latent heat per unit mass of the material currently converted, and $V$ is its volume. Equation (3) states that at the interface $S_{f}$ separating the phases heat is liberated or absorbed at a rate proportional to the volumetric rate of conversion of the material, $\mathrm{d} V / \mathrm{d} t$, and that this heat must be balanced by the heat flow $\Delta q^{S}$ from the interface. In equation (3) the plus sign is for the case of liberation of heat (solidification) and the minus sign is for the case of absorption of heat (melting).

In addition to the boundary conditions the initial temperature distribution must be given in a transient analysis.

\section{FINITE ELEMENT FORMULATION}

For finite element analysis it is necessary to write the governing equations in integral form, and then develop an incremental step-by-step solution. In the following exposition, we use the notation and procedures already defined in References 13-15. We assume that the conditions at time $t$ have been calculated, and that the temperatures are to be determined for the time $t+\Delta t$, where $\Delta t$ is the time increment. Using the Galerkin procedure and implicit time integration the heat flow equilibrium equations for the three-dimensional body considered at time $t+\Delta t$ are

$$
\begin{gathered}
\int_{V} \delta \theta^{\prime T t+\Delta t} \mathbf{k}^{t+\Delta t} \boldsymbol{\theta}^{\prime} \mathrm{d} V={ }^{t+\Delta t} Q+\int^{S_{c}} d \theta^{S t+\Delta t} h\left({ }^{t+\Delta t} \theta_{e}-{ }^{t+\Delta t} \theta^{S}\right) \mathrm{d} S \\
+\int_{S_{r}} \delta \theta^{S t+\Delta t} \kappa\left({ }^{t+\Delta t} \theta_{r}-{ }^{t+\Delta t} \theta^{S}\right) \mathrm{d} S
\end{gathered}
$$


where the superscript $t+\Delta t$ denotes 'at time $t+\Delta t$ ', $\delta$ means 'arbitrary variation in the temperatures that satisfy the temperature boundary conditions',

$$
\boldsymbol{\theta}^{T}=\left[\begin{array}{lll}
\frac{\partial \theta}{\partial x_{1}} & \frac{\partial \theta}{\partial x_{2}} & \frac{\partial \theta}{\partial x_{3}}
\end{array}\right]
$$

and, using conductivity principal co-ordinate directions,

$$
\mathbf{k}=\left[\begin{array}{ccc}
k_{11} & 0 & 0 \\
0 & k_{22} & 0 \\
0 & 0 & k_{33}
\end{array}\right]
$$

In equation (4), $S_{c}$ and $S_{r}$ are the surface areas with convection and radiation boundary conditions, respectively, and ${ }^{t+\Delta t} Q$ is the 'virtual work of the external heat flow input to the system at time $t+\Delta t^{\prime}$. The quantity ${ }^{t+\Delta t} Q$ includes the effects of surface heat flow inputs, ${ }^{t+\Delta t} q^{S}$, internal heat generation, ${ }^{t+\Delta t} q^{B}$, temperature-dependent heat capacity, ${ }^{t+\Delta t} c$, and latent heat effects, ${ }^{t+\Delta t} Q_{l}$. Hence

$$
{ }^{i+\Delta i} Q=\int_{S_{q}} \delta \theta^{S t+\Delta t} q^{S} \mathrm{~d} S+\int_{V} \delta \theta\left({ }^{t+\Delta t} q^{B}-\rho{ }^{t+\Delta t} c^{t+\Delta t} \dot{\theta}\right) \mathrm{d} V+{ }^{t+\Delta i} Q_{l}
$$

Equation (4) is a nonlinear equation in the unknown temperatures of the body. The equation is solved effectively by linearizing it about the conditions at time $t$, and then discretizing the incremental equations using isoparametric finite element procedures. ${ }^{13,15}$ Considering a given finite element mesh, the resulting finite element equations are in a modified Newton-Raphson iteration

$$
\left({ }^{t} \mathbf{K}^{k}+{ }^{t} \mathbf{K}^{c}+{ }^{t} \mathbf{K}^{r}\right) \Delta \boldsymbol{\theta}^{(i)}={ }^{t+\Delta t} \mathbf{Q}^{(i)}+{ }^{t+\Delta t} \mathbf{Q}^{c(i-1)}+{ }^{t+\Delta t} \mathbf{Q}^{r(i-1)}-{ }^{t+\Delta t} \mathbf{Q}^{k(i-1)}
$$

where ${ }^{t} \mathbf{K}^{k},{ }^{\prime} \mathbf{K}^{c}$ and ${ }^{t} \mathbf{K}^{r}$ are the conductivity, convection and radiation matrices, respectively; and the vectors ${ }^{t+\Delta t} \mathbf{Q}^{c(i-1)},{ }^{t+\Delta t} \mathbf{Q}^{r(i-1)}$ and ${ }^{t+\Delta t} \mathbf{Q}^{k(i-1)}$ are the nodal point heat flow vectors corresponding to convection, radiation and conduction effects, respectively. Also, the nodal point temperatures are given by

$$
\begin{gathered}
{ }^{t+\Delta t} \boldsymbol{\theta}^{(i)}={ }^{t+\Delta t} \boldsymbol{\theta}^{(i-1)}+\Delta \boldsymbol{\theta}^{(i)} \\
{ }^{t+\Delta t} \boldsymbol{\theta}^{(i)}={ }^{t} \boldsymbol{\theta}+\boldsymbol{\theta}^{(i)} \quad \boldsymbol{\theta}^{(i)}=\sum_{i=1}^{i} \Delta \boldsymbol{\theta}^{(j)}
\end{gathered}
$$

and the nodal point heat flow input vector is

$$
{ }^{i+\Delta t} \mathbf{Q}^{(i)}=\int_{V} \mathbf{H}^{T++\Delta t} q^{B} \mathrm{~d} V+\int_{S_{\mathbf{q}}} \mathbf{H}^{S^{T} t+\Delta t} q^{S} \mathrm{~d} S-\left(\int_{V} \rho{ }^{t+\Delta t} c^{(i-1)} \mathbf{H}^{T} \mathbf{H} \mathrm{d} V\right)^{t+\Delta t} \dot{\boldsymbol{\theta}}^{(i)}+{ }^{t+\Delta t} \mathbf{Q}^{(i-1)}
$$

where ${ }^{t+\Delta t} \mathbf{Q}_{i}^{(i-1)}$ is a result of the latent heat effects and is calculated as described in the following section.

The modified Newton-Raphson iteration in equation (6) has been used extensively without convergence difficulties, but the iteration may be accelerated using a full Newton or quasiNewton iteration. ${ }^{16}$

Considering equation (6) with ${ }^{i+\Delta t} \mathbf{Q}_{i}^{(i-1)}$ not included we note that these equilibrium relations are the usual equations solved in finite element analysis of nonlinear heat transfer. The equation can be solved effectively using the $\alpha$-method of time integration. ${ }^{13-15}$. We discuss in the next section how ${ }^{t+\Delta t} \mathbf{Q}_{i}^{(i-1)}$ is evaluated to include in a simple and effective manner the effects of phase changes when Euler backward integration (i.e. $\alpha=1$ ) is utilized. 


\section{EFFECT OF PHASE CHANGE}

The essence of our procedure is to construct the latent heat flow vector in equation (8) using the enthalpy of the system

$$
{ }^{t+\Delta t} H=\int_{V}\left\{\int_{0}^{t+\Delta t} \rho^{\tau} c^{\tau} \dot{\theta} \mathrm{d} \tau+\int_{0}^{t+\Delta t} \rho \dot{L} \mathrm{~d} \tau\right\} \mathrm{d} V
$$

Typical relationships between the enthalpy and temperature are shown schematically in Figure 2. In our scheme, the heat flow due to latent heat at node $k,{ }^{t+\Delta t} Q_{l, k}^{(i-1)}$, is calculated by defining $Q_{l, \text { total }, k}$ to be total latent heat flow available at node $k$ (where $Q_{t, \text { total, } k}$ is $\rho L / \Delta t$ integrated over the contributory nodal volume), $\theta_{k}^{(i)}$ to be the $i$ th approximation to the total temperature increment at node $k$ from ${ }^{t} \theta_{k}$ to ${ }^{t+\Delta t} \theta_{k}$, and considering pure substances and alloys as follows:

At the beginning of each time (load) step $i=1,{ }^{t+\Delta t} Q_{l, k}^{(0)}=0$

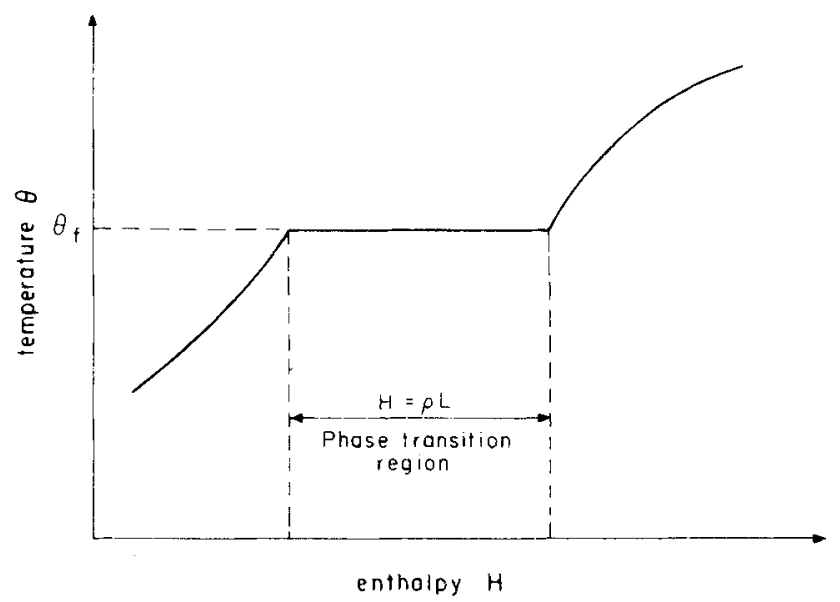

Figure 2(a). Enthalpy-temperature relationship for a pure substance with $\Delta \theta_{f}=0$

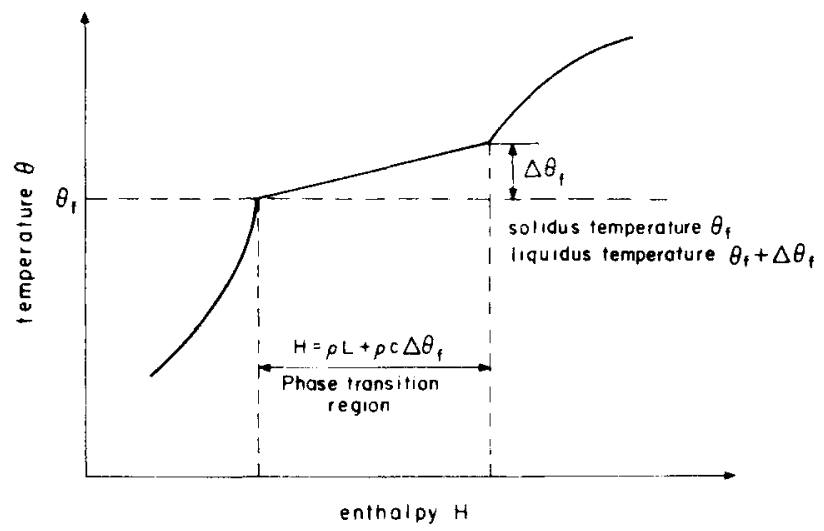

Figure 2(b). Enthalpy-temperature relationship for an alloy with phase transition temperature interval greater than zero. The latent heat $L$ and constant heat capacity $c$ are defined to correspond to the total enthalpy increment during phase transformation 
Pure substance $\left(\Delta \theta_{f}=0\right)$

Case (a); temperature is outside phase change:

$$
{ }^{t} \theta_{k}<\theta_{f} \text { and }{ }^{t+\Delta t} \theta_{k}^{(i)}<\theta_{f}
$$

or

$$
{ }^{t} \theta_{k}>\theta_{f} \text { and }{ }^{t+\Delta t} \theta_{k}^{(i)}>\theta_{f}
$$

then

$$
\begin{aligned}
\bar{\theta}_{k}^{(i)} & =\theta_{k}^{(i)} \\
\Delta Q_{l, k}^{(i)} & =0
\end{aligned}
$$

Case (b); temperature passes through phase change temperature:

$$
{ }^{\mathrm{s}} \theta_{k}=\theta_{f}
$$

or

$$
{ }^{t} \theta_{k}<\theta_{f} \text { and }{ }^{i+\Delta_{t}} \theta_{k}^{(i)} \geqslant \theta_{f}
$$

or

$$
{ }^{t} \theta_{k}>\theta_{f} \text { and }{ }^{t+\Delta t} \theta_{k}^{(i)} \leqslant \theta_{f}
$$

then

$$
\begin{gathered}
\bar{\theta}_{k}^{(i)}=\theta_{f}-{ }^{t} \theta_{k} \\
\Delta Q_{l, k}^{(i)}=-\int_{V_{k}} \frac{1}{\Delta t} \rho^{i+\Delta t} c^{(i-1)}\left(\theta_{k}^{(i)}-\bar{\theta}_{k}^{(i)}\right) \mathrm{d} V
\end{gathered}
$$

where the volume integration is performed over the volume $V_{k}$ associated (in a finite element sense) with node $k$, until

$$
\sum \Delta Q_{l, k}^{(i)}= \pm Q_{l, \text { total }, k}
$$

where we sum over all iterations (and + is for solidification, while - is for melting) and for cases (a) and (b) we have

$$
\begin{aligned}
{ }^{t+\Delta t} \theta_{k}^{(i)} & ={ }^{t} \theta_{k}+\bar{\theta}_{k}^{(i)} \\
{ }^{t+\Delta t} Q_{l, k}^{(i)} & ={ }^{t+\Delta t} Q_{l, k}^{(i-1)}+\Delta Q_{l, k}^{(i)}
\end{aligned}
$$

Alloy substance $\left(\Delta \theta_{f}>0\right)$

Case (a); temperature is outside phase change temperature interval:

$$
{ }^{t} \theta_{k}<\theta_{f} \text { and }{ }^{t+\Delta t} \theta_{k}^{(i)}<\theta_{f}
$$

or

$$
{ }^{i} \theta_{k}>\theta_{f}+\Delta \theta_{f} \text { and }{ }^{i+\Delta ;} \theta_{k}^{(i)}>\theta_{f}+\Delta \theta_{f}
$$

then

$$
\begin{aligned}
\bar{\theta}_{k}^{(i)} & =\theta_{k}^{(i)} \\
\Delta Q_{l, k}^{(i)} & =0
\end{aligned}
$$


Case (b); temperature passes through phase change temperature interval:

$$
\theta_{f} \leqslant \theta_{k} \leqslant \theta_{f}+\Delta \theta_{f}
$$

or

$$
{ }^{t} \theta_{k}<\theta_{f} \text { and }{ }^{t+\Delta t} \theta_{k}^{(i)} \geqslant \theta_{f}
$$

or

$$
{ }^{t} \theta_{k}>\theta_{f}+\Delta \theta_{f} \quad \text { and } \quad{ }^{t+\Delta t} \theta_{k}^{(i)} \leqslant \theta_{f}+\Delta \theta_{f}
$$

then

$$
\Delta Q_{l, k}^{(i)}=-\int_{V_{k}} \frac{1}{\Delta t} \rho c^{*}\left(\theta_{k}^{(i)}-\theta_{f}+{ }^{t} \theta_{k}\right) \mathrm{d} V
$$

where the volume integration is performed over the volume $V_{k}$ associated (in a finite element sense) with node $k$, and

$$
\begin{gathered}
c^{*}=\frac{1}{\left(\frac{\Delta \theta_{f}}{L}\right)+\left(\frac{1}{t+\Delta t} c^{(i-1)}\right)} \\
\bar{\theta}_{k}^{(i)}=\theta_{f}-{ }^{t} \theta_{k}+\left[\frac{\sum \Delta Q_{l, k}^{(i)}}{Q_{l, \text { total }, k}}\right] \Delta \theta_{f}
\end{gathered}
$$

until

$$
\sum \Delta Q_{l, k}^{(i)}= \pm Q_{l, \text { total }, k}
$$

where we sum over all iterations (and + is for solidification, while - is for melting) and it should be noted that the specific heat is assumed constant (as defined in Figure $2 b$ ) during phase transformation. For cases (a) and (b) we have

$$
\begin{aligned}
{ }^{1+\Delta t} \theta_{k}^{(i)} & ={ }^{i} \theta_{k}+\bar{\theta}_{k}^{(i)} \\
{ }^{t+\Delta t} Q_{l, k}^{(i)} & ={ }^{t+\Delta t} Q_{l, k}^{(i-1)}+\Delta Q_{l, k}^{(i)}
\end{aligned}
$$

The algorithm for alloy substances reduces to the algorithm for pure substances when $\Delta \theta_{f}=0$. These constraints enforce nodal temperatures passing through the phase transition to reach a temperature consistent with the amount of latent heat liberated or absorbed. Our procedure is quite simply implemented using a diagonal heat capacity matrix (which uncouples the nodal enthalpies), a vector for accumulating latent heat liberated or absorbed, and a vector storing the total latent heat available at each node.

It should be noted that both the heat flow balance and enthalpy conditions are explicitly imposed in this formulation. The finite element mesh and time step size for a specific analysis can therefore largely be chosen by considering the accuracy predicted on the temperatures when phase changes are neglected. In particular, as noted in the sample solutions, the phase change front may advance over several elements in a single time step, and the phase front location is predicted accurately with a relatively coarse finite element mesh and relatively large time steps.

\section{SAMPLE SOLUTIONS}

We have incorporated our algorithm for predicting the propagation of a phase change front into the ADINAT computer program ${ }^{14}$ and analysed a variety of problems. In the following, 
we present the solution of two test cases analysed previously by a number of researchers. In the first two analyses $\Delta \theta_{f}=0$ which represents a physical condition not readily modelled with the algorithm of Morgan et al..$^{8}$ In the third analysis $\Delta \theta_{f}=10^{\circ} \mathrm{F}$. In all analyses the Euler backward time integration scheme $(\alpha=1)$ and lumped heat capacity matrices were used. ${ }^{13}$

\section{Solidification of a semi-infinite slab of liquid}

The uniform infinite slab of liquid is considered initially at zero temperature as shown in Figure 3. At time $t=0^{+}$, the temperature of the surface of the liquid is reduced to $-45^{\circ} \mathrm{F}$ and maintained constant. The conductivity, specific heat and density of the liquid and solid phases are assumed equal and constant. The problem was, for example, considered earlier by G. Comini et al., ${ }^{8}$ Morgan et al. ${ }^{9}$ and Ichikawa and Kikuchi. ${ }^{11}$

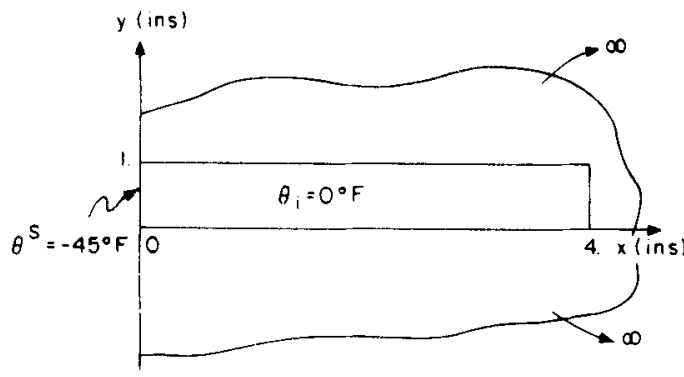

$$
\begin{aligned}
& \text { MATERIAL PROPERTIES: } \\
& k=1.08 \text { B } \mathrm{tu} / \mathrm{in} \mathrm{sec}{ }^{\circ} \mathrm{F} \\
& \rho C=1 . \quad 8+\mathrm{w} / \mathrm{in}^{3}-{ }^{\circ} \mathrm{F} \\
& \rho L=70.26 \mathrm{~B}+\mathrm{u} / \mathrm{in}^{3} \\
& \theta_{\mathrm{f}}=-1 \quad \text { OF }
\end{aligned}
$$

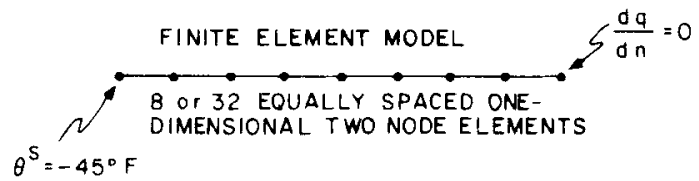

Figure 3. Model for analysis of the solidification of a semi-infinite slab of liquid

In our analysis we used different finite element models and time steps. Figures 4 and 5 show the 8 and 32 element solutions, respectively, when using different time steps, $\Delta t$. It is noted that in all analyses the freezing front position is very accurately predicted, even with the 8-element model and the time step $\Delta t=1 \mathrm{sec}$. However, for an accurate prediction of the temperature, a finer finite element discretization and smaller time step $\Delta t$ need be employed. The temperature oscillations obtained are similar to those observed in the analysis of wave propagation problems, and are due to the fact that all latent heat for the material lumped at a node must be released or absorbed before the nodal temperature is allowed to vary. We should also note that, in the analyses, the phase change front advanced over a number of elements per time step. 

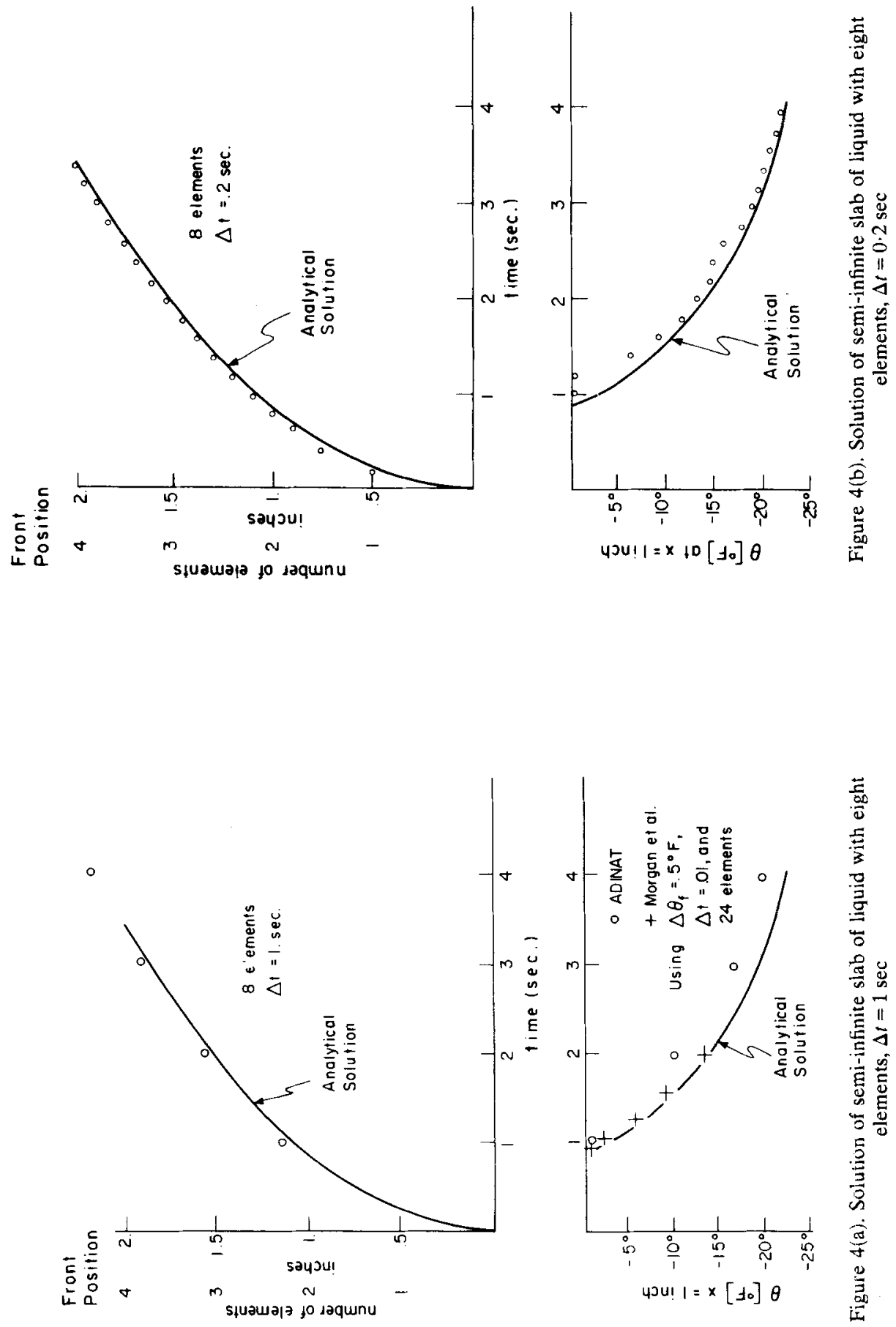

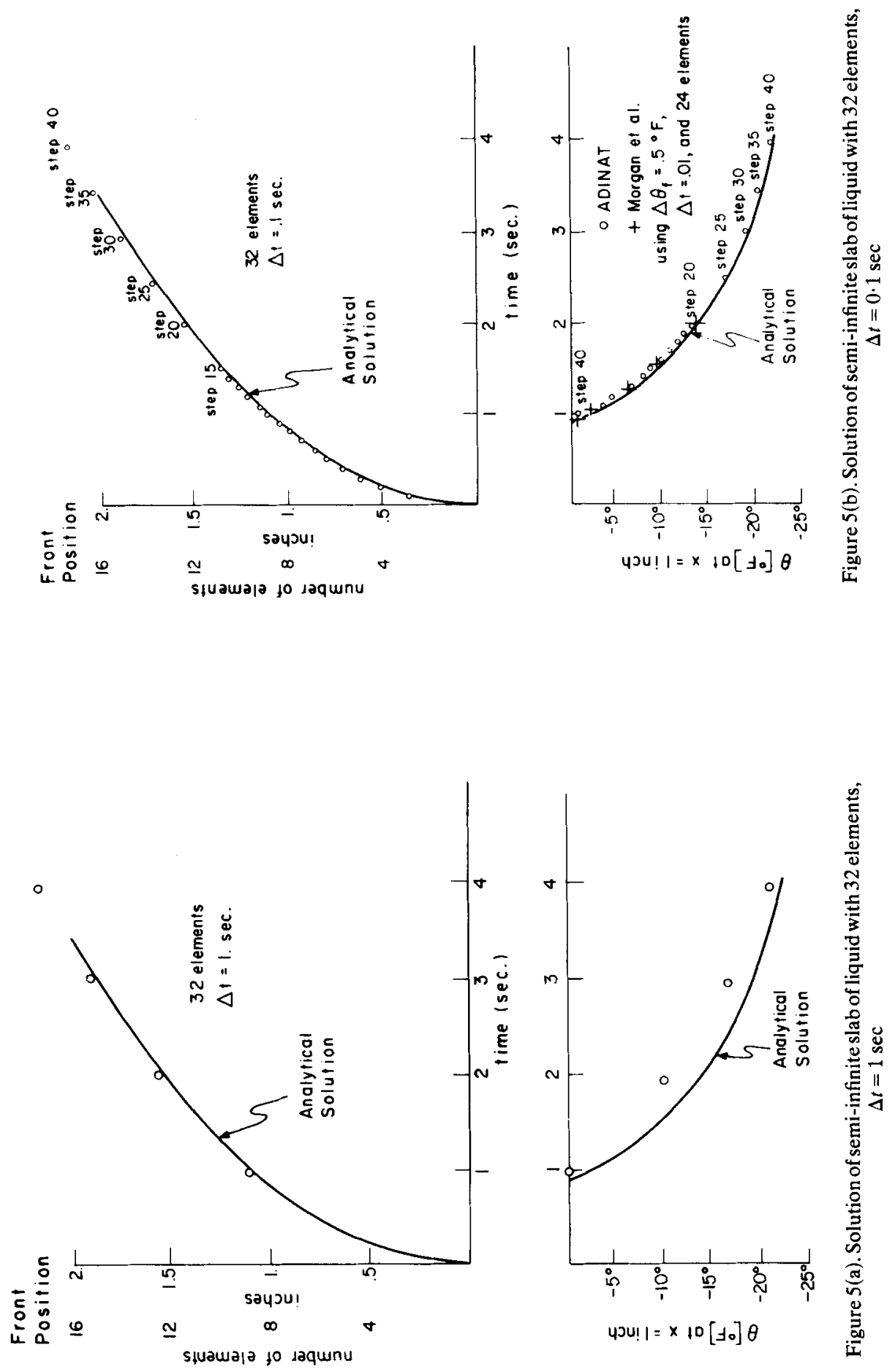


\section{Solidification of a corner region}

The corner of a uniform infinite container carrying a liquid with initial temperature $\theta_{i}$ and freezing temperature $\theta_{f}$ is considered (see Figure 6). At time $t=0^{+}$, the temperature of the surface, $\theta^{S}$, is reduced to a temperature lower than the freezing temperature and is maintained constant. The thermal conductivity, specific heat and density of the liquid and solid phases are assumed equal and constant. The solution for the temperature and the position of the

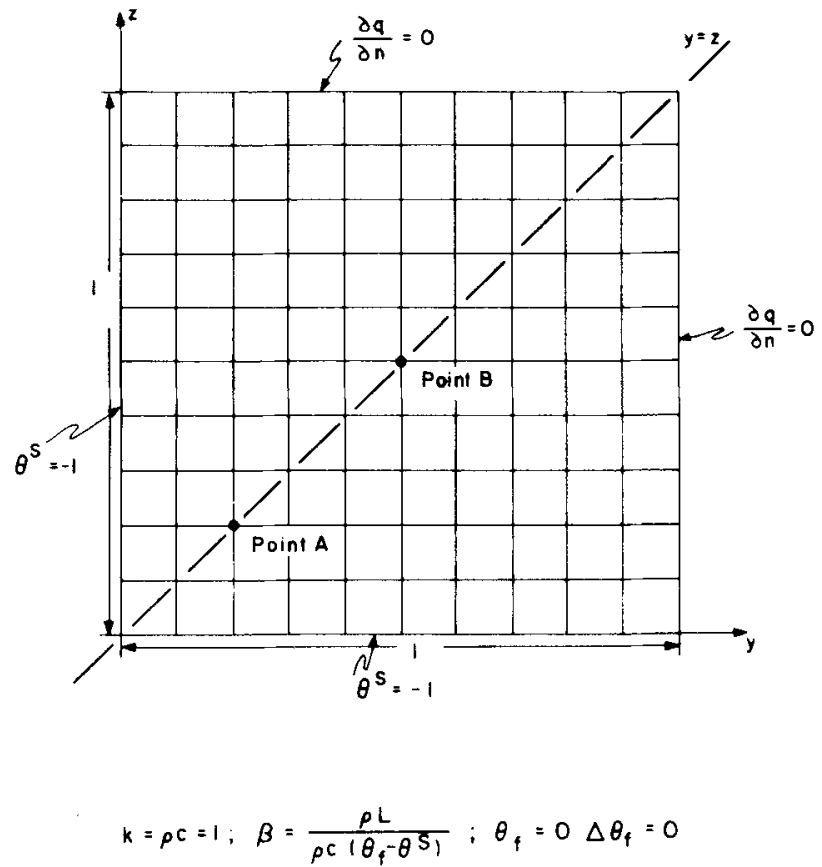

Figure 6. Model for solution of solidification of corner region

phase transition surface depend upon the values of $\theta^{s}, \theta_{i}$ and $\beta$ assumed. The values assumed in the two analyses performed are:

1. Liquid initially at the freezing temperature:

$$
\theta^{S}=-1 \quad \theta_{i}=0 \quad \beta=1.5613
$$

2. Liquid initially at a temperature higher than freezing temperature:

$$
\theta^{S}=-1 \quad \theta_{i}=0.3 \quad \beta=0 \cdot 25
$$

This problem was analysed previously by Budhia and Krieth, ${ }^{6}$ Comini et al., ${ }^{8}$ Morgan et al. ${ }^{9}$ and Ichikawa and Kikuchi. ${ }^{1}$

Figures 7-10 give the solutions obtained. We note that the same basic observations regarding the performance of our algorithm are applicable as in the solution of the solidification of the slab of liquid (previous section). 

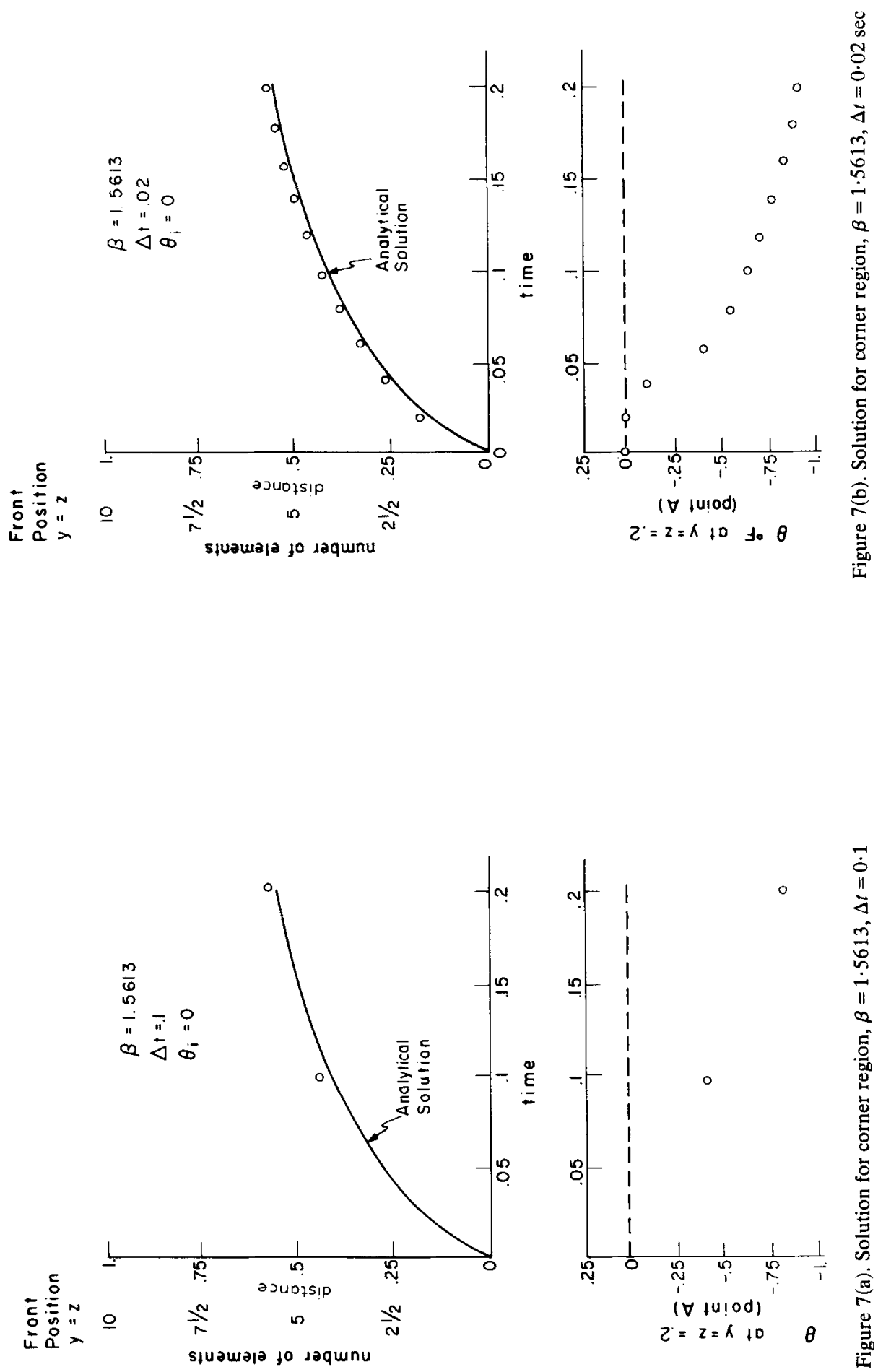


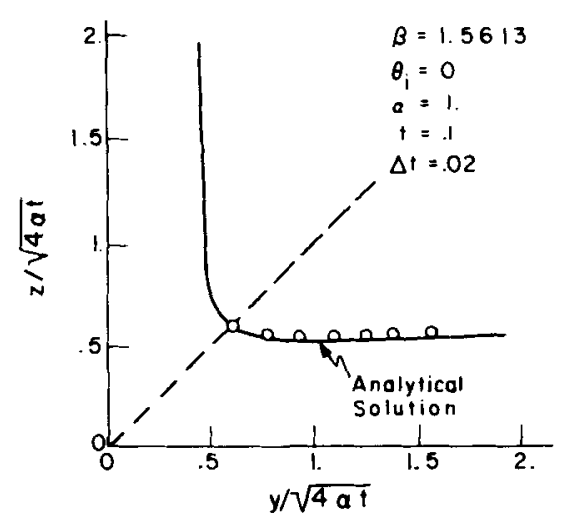

(a)

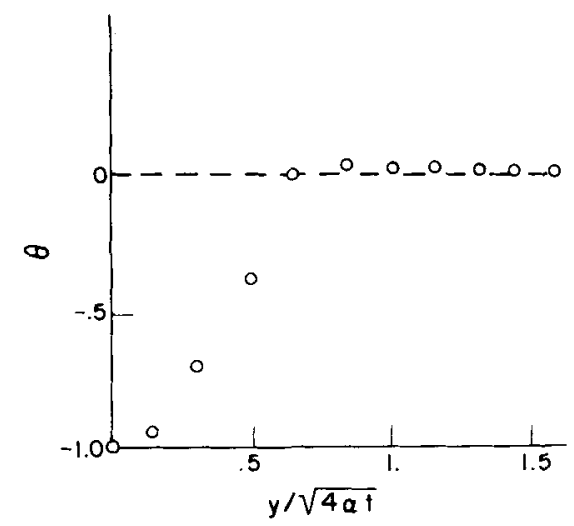

(b)

Figure 8. Solidification of a corner region, $\beta=1 \cdot 5613$ : (a) non-dimensional phase front position; (b) non-dimensional temperature profile along $y=z$

Solidification of a semi-infinite slab of liquid with $\Delta \theta_{f}=10^{\circ} \mathrm{F}$

This problem is essentially identical to the problem described earlier and in Figure 3, with the exception that the phase transition temperature interval is now $10^{\circ} \mathrm{F}$. Two models were analysed using ADINAT; a model of eight equal elements with a time step $\Delta t=0.2 \mathrm{sec}$ and a model of 32 equal elements with a time step $\Delta t=0 \cdot 1 \mathrm{sec}$. The temperature response predicted at $x=1$ in. into the slab using these models is shown in Figure 11.

An analytical solution to this problem could not be established and, therefore, an alternative numerical solution based on the integration of the enthalpy as suggested by Atthey and by Levy ${ }^{17,18}$ was performed. In this solution the basic equations used were

$$
\begin{aligned}
{ }^{t+\Delta t} \mathbf{H} & ={ }^{t} \mathbf{H}+{ }^{t} \mathbf{Q} \Delta t-{ }^{t} \mathbf{K}^{t} \boldsymbol{\theta} \Delta t \\
{ }^{t+\Delta t} \boldsymbol{\theta} & =f\left({ }^{t+\Delta t} \mathbf{H}\right)
\end{aligned}
$$

where the temperatures are obtained from the temperature-enthalpy relation (Figure 2). Figure 11 also shows the response predicted in this alternative numerical solution with 64 elements and a time step $\Delta t=0.0001 \mathrm{sec}$. We observe that the results predicted with our algorithm in ADINAT using 32 elements and a time step $\Delta t=0 \cdot 1 \mathrm{sec}$, are close to those obtained by integration of the enthalpy. 

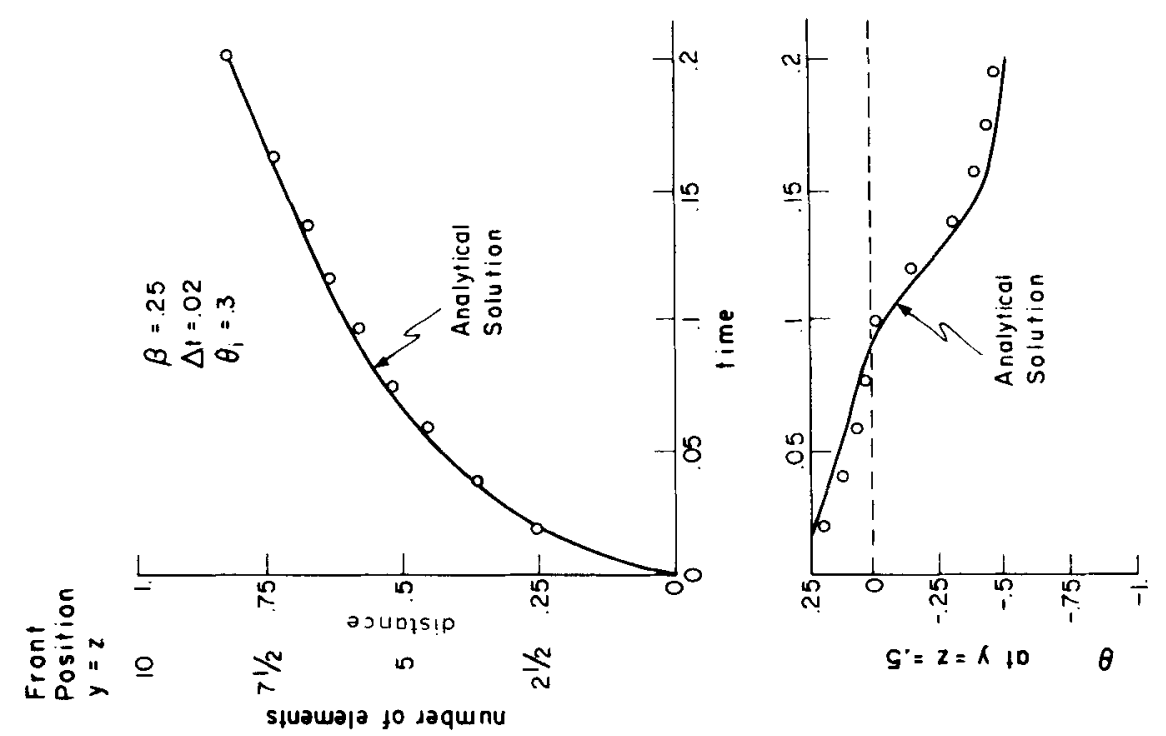

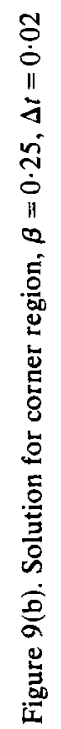
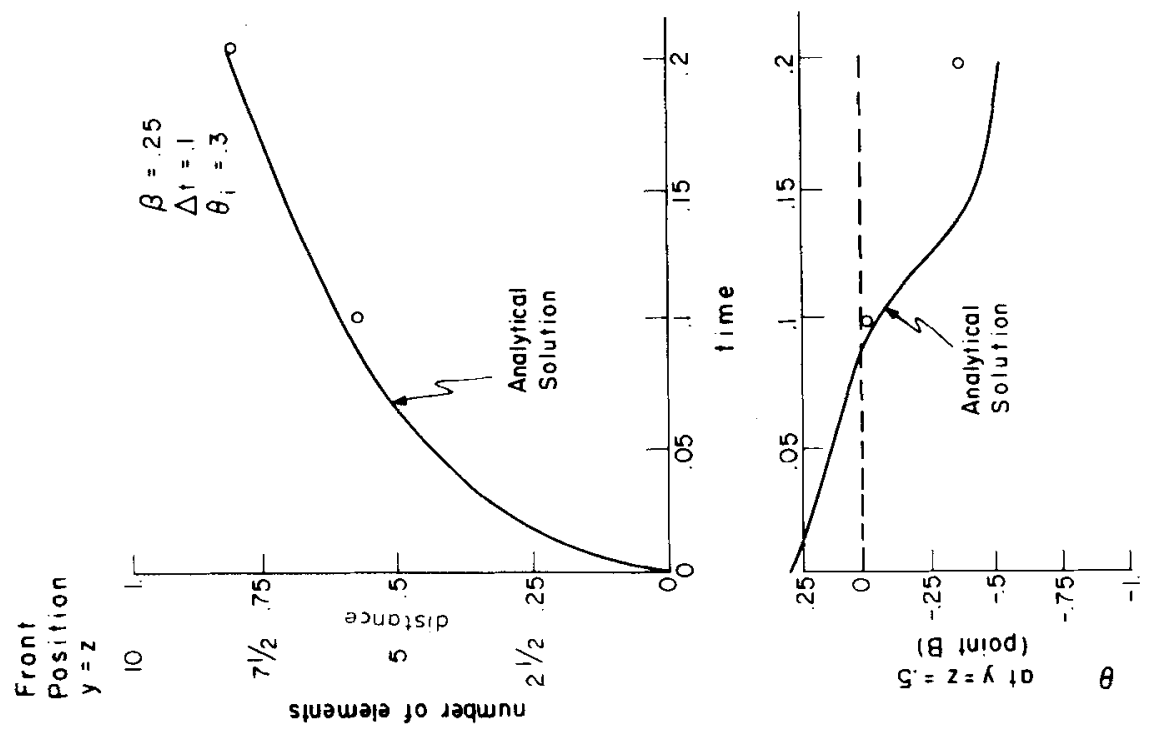

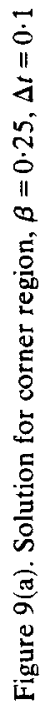



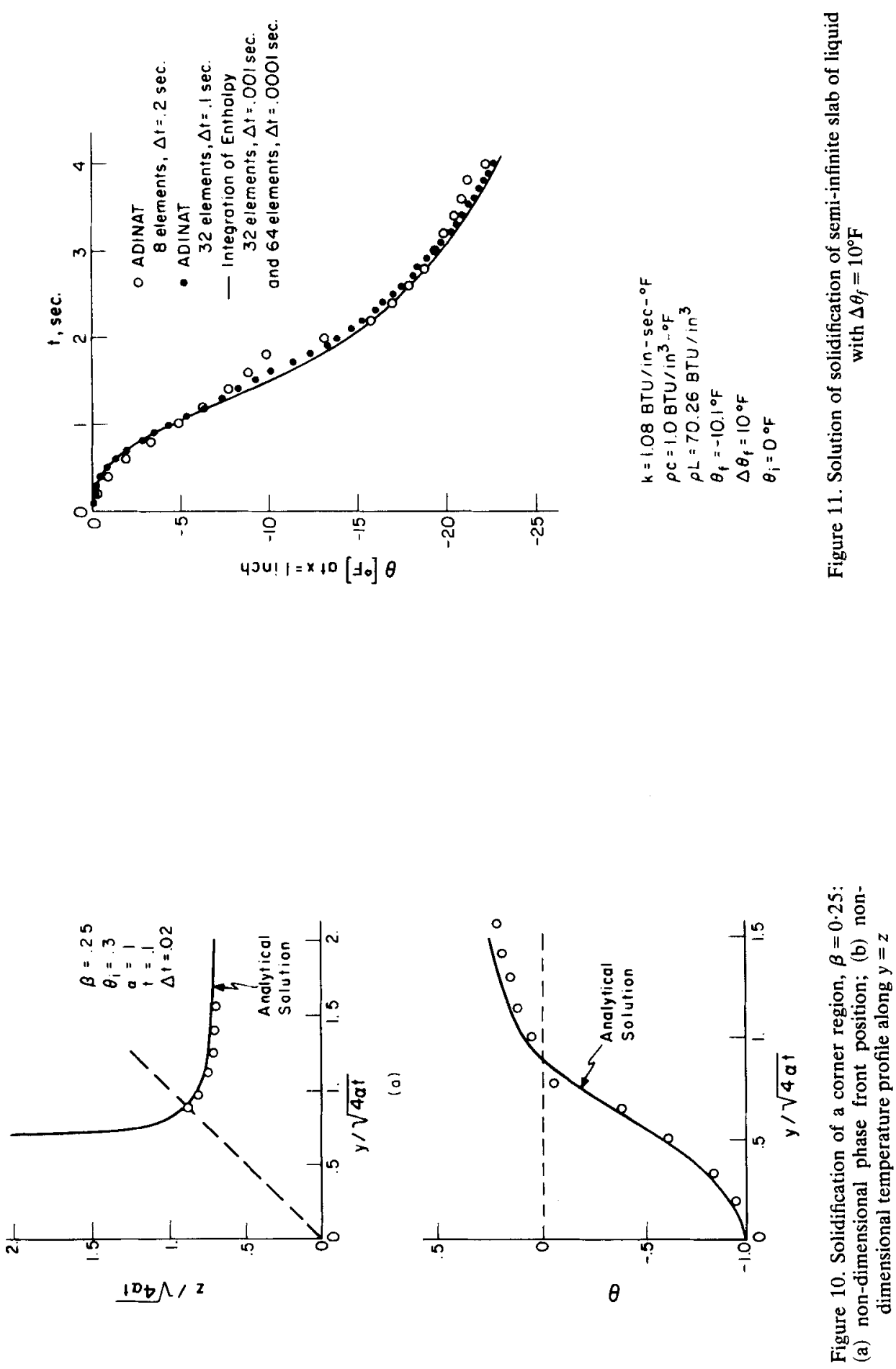


\section{CONCLUSIONS}

A simple and effective procedure for the analysis of nonlinear transient thermal problems including phase change effects has been presented. The procedure is based on iterating with both the enthalpy and the heat flow in the phase transition region. The method allows the use of a relatively coarse finite element mesh and relatively large time steps, and allows the use of a phase transition temperature interval of zero, for a pure substance, or greater than zero for an alloy. Our current experience with the algorithm indicates that the finite element mesh and time step size for a specific analysis including phase changes can largely be the same as those used in the analysis of the problem neglecting phase change effects. However, a detailed theoretical analysis of the algorithm would be very valuable. The procedure is readily incorporated into existing finite element computer programs for nonlinear heat transfer analysis.

\section{ACKNOWLEDGEMENTS}

We would like to thank Sam Levy, General Electric Corporation R. and D., Schenectady, New York, for some stimulating discussions during the course of this research. We are grateful for the financial support of this work by the GKSS Forschungszentrum, West Germany and the ADINA users' group.

\section{REFERENCES}

1. J. Stefan, 'Über die Theorie der Eisbildung, Insbesondere Über die Eisbildung in Polarmeere', Ann. Phys. Chem., 42, 269-286 (1891).

2. J. A. Wheeler, 'Simulation of heat transfer from a warm pipeline buried in permafrost', Esso Production Research Co., presented at 74th Nat. Mtg A.I.Ch.E., New Orleans, Louisiana, March 11-15, 1973.

3. H. D. Hibbitt and P. V. Marcal, 'A numerical thermomechanical model for the welding and subsequent loading of a fabricated structure', Computers Struct., 3, 1145-1174 (1973).

4. A. Friedman, 'The Stefan problem in several space variables', Trans. Amer. Math. Soc., 133, 51-87 (1968).

5. G. H. Meyer, 'Multidimensional Stefan problems', S.I.A.M. J. Numer. Anal., 10, 522-538 (1973).

6. H. Budhia and F. Krieth, 'Heat transfer with melting or freezing in a wedge', Int. J. Heat Mass Trans., 16, 195-211 (1973)

7. D. R. Atthey, 'A finite difference scheme for melting problems', J. Inst. Math. Applics., 13, 353-366 (1974).

8. G. Comini, S. Del Guidice, R. W. Lewis and O. C. Zienkiewicz, 'Finite element solution of non-linear heat conduction problems with special reference to phase change', Int. J. num. Meth. Engng, 8, 613-624 (1974).

9. K. Morgan, R. W. Lewis and O. C. Zienkiewicz, 'An improved algorithm for heat conduction problems with phase change', Int. J. num. Meth. Engng, 12, 1191-1195 (1978).

10. J. T. Oden and N. Kikuchi, 'Finite elememt methods for certain free boundary-value problems in mechanics', in Moving Boundary Problems (D. G. Wilson, A. D. Solomon and P. T. Boggs, Eds.), Academic Press, 1978.

11. Y. Ichikawa and N. Kikuchi, 'A one-phase multi-dimensional Stefan problem by the method of variational inequalities', Int. J. num. Meth. Engng, 14, 1197-1120 (1979).

12. N. Kikuchi and Y. Ichikawa, 'Numerical methods for a two-phase Stefan problem by variational inequalities', Int. J. num. Meth. Engng, 14, 1221-1239 (1979).

13. K. J. Bathe and M. R. Khoshgoftaar, 'Finite element formulation and solution of nonlinear heat transfer', J. Nucl. Eng. Design, 51, 349-401 (1979).

14. K. J. Bathe, 'ADINAT-A finite element program for automatic dynamic incremental nonlinear analysis of temperatures', Report AVL 82448-5, Dept. of Mechanical Eng., M.I.T., May 1977 (rev. Dec. 1978).

15. K. J. Bathe, Finite Element Procedures in Engineering Analysis, Prentice-Hall, Inc., N.J., 1981.

16. K. J. Bathe and A. P. Cimento, 'Some practical procedures for the solution of nonlinear finite element equations', J. Comp. Meth. Appl. Mech. Eng., 22, 59-85 (1980).

17. D. R. Atthey, 'A finite difference scheme for melting problems based on the method of weak solutions', in Moving Boundary Problems in Heat Flow and Diffusion (J. R. Ockendon and W. R. Hodgkin, Eds.), Clarendon Press, Oxford, 1975.

18. S. Levy, General Electric Corporation R. and D., Schenectady, New York, personal communication on research efforts performed during late 1960 s. 